\title{
PREDICTION OF ACOUSTIC MODAL CHARACTERISTICS OF TWO-DIMENSIONAL IRREGULAR SHAPED CAVITIES BY IMPEDANCE MOBILITY COMPACT MATRIX (IMCM) APPROACH
}

\author{
VEERABHADRA REDDY \\ Research Center Imarat, Hyderabad, Telangana, India, and \\ Indian Institute of Technology Hyderabad, Department of Mechanical and Aerospace Engineering, Telangana, India \\ e-mail: me13p1005@iith.ac.in \\ B. Venkatesham \\ Indian Institute of Technology Hyderabad, Department of Mechanical and Aerospace Engineering, Telangana, India \\ e-mail:venkatesham@iith.ac.in \\ V. Narayana Murthy \\ Research Center Imarat, Hyderabad, Telangana, India \\ e-mail:v.narayanamurthy@rcilab.in
}

\begin{abstract}
In this paper, the impedance and mobility compact matrix (IMCM) method for prediction of acoustic modal characteristics of two-dimensional irregular cavities with the rigid wall boundary is presented. This method consists of discretizing the whole cavity into a series of subcavities either of regular or irregular shape. Continuity of both pressure and velocity between adjacent subcavities is ensured using a virtual membrane with zero mass and stiffness. Mathematical formulation for acoustic cavities with the irregular shape has been explained in detail. A finite element model has been developed to calculate the acoustic natural frequency and mode shape. The proposed method is validated using a regular and irregular cavity and compared with finite element modelling results and available results in the literature.
\end{abstract}

Keywords: impedance, mobility, acoustic modal characteristics, regular and irregular cavity

\section{Introduction}

Interior noise associated with vibrating structures has been the subject of concern in areas like Heating, Ventilation and Air-Conditioning (HVAC) systems, cabins, buildings, aircraft and vehicles. HVAC systems extensively used ducts of different size and shapes connected in series or parallel. Most prominent duct shapes are rectangular, circular, flat oval and elliptical. The coupling of acoustical and structural duct modes plays a critical role in generating noise in the transverse direction. The phenomenon of coupling is called the vibro-acoustic coupling. The first step in understanding the vibro-acoustic coupling is to know modal characteristics of these ducts. Prediction of modal characteristics of acoustic cavities with irregular shapes is an important aspect in vibro-acoustic analysis of systems. Modal characteristics include natural frequencies and acoustic mode shapes. Analytical expressions are available to calculate natural frequencies and mode shapes of cavities which are limited to regular and simple geometries, such as rectangular and cylindrical cavities. Various methods are available for prediction of modal characteristics of cavities with an arbitrary shape. These methods include Finite Element Method (FEM) (Petyt et al., 1976; Joppa and Fyfe, 1978), Boundary Element Method (BEM) (Brebbia et al., 1984), Acousto Elastic Method (AEM) (Dowell et al., 1977), Green's function method (GFM) (Morse and Feshbach, 1953), and Integro Modal Method (IMM) (Missaoui and 
Cheng, 1997). FEM and BEM are very popular and widely used for prediction of acoustic natural frequencies of arbitrary geometries; however they need a large number of degrees of freedom and computational time. AEM predicts modal characteristics of multiply connected regular or irregular shaped cavities by approximating cavity geometry by rectangular subcavities. Approximation of the geometry leads to errors in computation of modal characteristics. GFM is limited to a cavity slightly distorted from a regular one. IMM provides a combined approach of AEM and GFM retaining the advantages of both the methods. Few other research works include the point-matching method (Kang and Lee, 2000) and a method using wave guide type base functions (Kim and Kim, 1999; Amir and Starobinski, 1996) to compute the natural frequencies of arbitrarily shaped two-dimensional cavities. These studies have not emphasized computation of the corresponding acoustic mode shapes. Pan and Bies (1990) studied the effect of fluid structural coupling on sound waves in an enclosure using a rigid wall mode expansion and also discussed the limitations of the method. Pan (1999) introduced the concept of an extended mode shape function where the absorptive or flexible part of the cavity boundary was assumed. Anyunzoghe and Cheng (2002) improved the integro-modal approach developed previously and introduced the technique of overlapped subcavities for better convergence of the pressure gradient in the vicinity of the junctions between the subcavities. This method gives few spurious modes of the individual cavities in addition to the natural modes which are to be discarded. Kim and Brennan (1999) presented a compact matrix formulation for the steady-state analysis of a vibro-acoustic system. It is based on the impedance and mobility approach using the uncoupled mobility of the structure and uncoupled acoustic impedance, both in modal coordinate systems. This method is very effective for investigating coupling between structural and acoustic systems. Sum and Pan (2005) studied the effects of the inclination of a rigid wall on free vibration characteristics of acoustic modes in a trapezoidal cavity, and found that for small inclinations, each trapezoidal cavity mode possessed a distorted shape of the rectangular cavity mode that evolved it. When the inclination was increased, the trapezoidal cavity-mode shape became complicated and unrecognizable. Venkatesham et al. (2008) presented free vibration analysis of coupled acoustic-structural systems of rectangular ducts using the impedance-mobility compact matrix method (IMCM). The same method can be extended for free vibration analysis of irregular shapes by dividing into regular subcavities with a virtual membrane. Shi et al. (2019) proposed a method for analysis of acoustic modals and steady-state responses of an arbitrary triangular prism and a quadrangular prism acoustic cavities based on the three-dimensional improved Fourier series.

In this paper, an alternative approach based on the impedance and mobility compact matrix (IMCM) method is presented for prediction of acoustic modal characteristics of irregular cavities. This method will help us to cascade the subcavity impedances in a matrix form. Mathematical formulation and generalization of the method for acoustic cavities with irregular shapes has been explained in detail. A standard eigenvalue problem is then established. Numerical results for regular cavity and irregular cavity are then presented and compared with available results in the literature or with other existing methods.

\section{Impedance and Mobility Compact Matrix Method (IMCM)}

The proposed mathematical formulation based on the assumption of an irregular shaped cavity can be treated as a combination of connected regular and irregular shaped subcavities separated by virtual membranes. In each subcavity, the acoustic pressure $P$ can be calculated using the Kirchhoff-Helmholtz integral (Missaoui and Cheng, 1997)

$$
\int_{V} G\left(\mathbf{r}, \mathbf{r}_{0}\right)\left(\nabla^{2} P+k^{2} P\right) d v=\int_{V} P\left(\nabla^{2} G+k^{2} G\right) d v+\int_{S_{b}}\left(G\left(\mathbf{r}, \mathbf{r}_{0}\right) \frac{\partial P}{\partial n}-P \frac{\partial G}{\partial n}\right) d s
$$


where $k$ is the wave number, $n$ is the outward normal vector of the enclosed boundary surface $S_{b}$ with the volume $V, \mathbf{r}, \mathbf{r}_{0}$ are the observation and source point, respectively, $G$ is the Green function for the interior cavity domain, and is given in terms of mode shapes as (Missaoui and Cheng, 1997)

$$
G\left(\mathbf{r}, \mathbf{r}_{0}\right)=\sum_{n} \frac{c^{2} \varphi_{n}(\mathbf{r}) \varphi_{n}\left(\mathbf{r}_{0}\right)}{\left(\omega_{n}^{2}-\omega^{2}\right) \Lambda_{n}}
$$

where $c$ is the speed of sound in the internal medium, $\omega_{n}$ is the angular natural frequency of the cavity, $\varphi_{n}$ is the corresponding mode shape and $\Lambda_{n}$ is the normalised acoustic mass of the cavity

$$
\Lambda_{n}=\int_{V} \varphi_{n}^{2}(\mathbf{r}) d v
$$

Figure 1a shows a typical two-dimensional irregular cavity. This cavity is divided into a series of regular shapes like rectangular, cylindrical, or semi-cylindrical and irregular subcavities as shown in Fig. 1b. These subcavities are connected by a virtual membrane with zero mass and stiffness at the junctions. Irregular subcavities are bounded by regular boundaries, $S_{d}$ is the surface of irregular subcavities, $n_{d}$ is the normal to the irregular surface, $n_{r}$ is the normal to the surface at the junctions. The discretised subcavity numbers may be chosen based on the convergence criteria for the acoustical modal characteristics. However, four subcavities are chosen for demonstration of the proposed methodology. An irregular shaped subcavity in domain 2, 3 is a deviation from the bounded regular shape as shown in Fig. 1b. Mode shapes and natural frequencies of these subcavities cannot be estimated by analytical expressions like regular-shaped subcavities in domain 1 and 4 . To estimate the mode shapes and natural frequencies of irregular shaped cavities, as shown in Fig. 1a, the procedure consists of enclosing the irregular subcavity by a regular one, called the enveloping or bounding subcavity for which modal information is available.

(a)

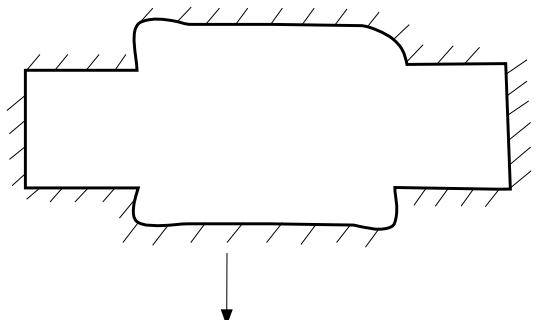

(b)

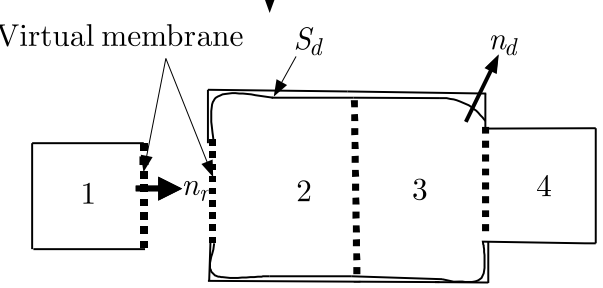

Fig. 1. (a) Schematic of irregular cavity. (b) Discretization of irregular cavity with regular and irregular subcavities with virtual membrane junctions

The acoustic pressure $P(\mathbf{r}, \omega)$ inside the subcavity and vibration velocity of the virtual membrane $u(\mathbf{r}, \omega)$ are expressed in terms of a finite number of mode shape functions as (Kim and Brennan, 1999)

$$
P(\mathbf{r}, \omega)=\sum_{n=1}^{N} \varphi_{n}(\mathbf{r}) a_{n}(\omega)=\boldsymbol{\Phi}^{\mathrm{T}} \mathbf{a} \quad u(\mathbf{r}, \omega)=\sum_{m=1}^{M} \psi_{m}(\mathbf{r}) b_{m}(\omega)=\mathbf{\Psi}^{\mathrm{T}} \mathbf{b}
$$


where $n$ (consisting of $n_{x}, n_{y}$ ) is an acoustic modal index, $N$ represents the finite number of acoustic modes, $m$ is a structural modal index and $M$ represents the finite number of structural modes. The $N$ length column vectors $\boldsymbol{\Phi}$ and a consists of an array of uncoupled acoustic mode shape functions for the rigid wall cavities $\left(\varphi_{n}(\mathbf{r})\right)$ and the amplitude of the acoustic pressure mode $a_{n}(\omega)$, respectively. Similarly, the $M$ length column vectors $\boldsymbol{\Psi}$ and $\mathbf{b}$ consists of an array of uncoupled vibration mode shape functions of the virtual membrane $\psi_{m}(\mathbf{r})$ and the uncoupled amplitude of the vibration velocity modes $b_{m}(\omega)$, respectively. Equations (2.2)-(2.4) are substituted in Eq. (2.1) to obtain the amplitude of the $n$-th acoustic mode in irregular cavities, and it is written as

$$
a_{n}(\omega)=\frac{\rho_{0} c_{0}^{2}}{\Lambda_{n}} A_{n}(\omega)\left(q_{n}+\sum_{m=1}^{M} C_{n, m} b_{m}(\omega)-\frac{1}{j \omega} \sum_{n=1}^{N} \frac{a_{n^{\prime}}}{\Lambda_{n^{\prime}}} D_{n, n^{\prime}}\right)
$$

where $q_{n}$ is the generalised acoustic source strength, $A_{n}(\omega)$ is the acoustic mode resonance term, $\rho_{0}$ is density of the air, and $C_{n, m}$ represents the geometrical coupling relationship between the uncoupled structural and acoustic mode shape functions on the surface of the virtual membrane $S_{f}$, and is given by

$$
C_{n, m}=\int_{S_{f}} \varphi_{n}(\mathbf{r}) \psi_{m}(\mathbf{r}) d s
$$

$D_{n, n^{\prime}}$ is the spatial coupling between the $n$-th and $n^{\prime}$-th acoustic modes of the bounding cavity over the irregular surface $S_{d}$, and is given by

$$
D_{n, n^{\prime}}=\int_{S_{d}} \phi_{n^{\prime}} \frac{\partial \phi_{n}}{\partial n_{d}} d s
$$

In Eq. (2.7), integration is performed over the surface of the irregular cavity, either analytically or numerically. Any irregularity of the boundary shape has some effect on coupling the acoustic modes of its envelope. However, for regular subcavities as $\phi_{n^{\prime}}=\phi_{n}$, then $D_{n, n^{\prime}}=0$.

Assuming that damping is negligible for modal analysis

$$
A_{n}(\omega)=\frac{j \omega}{\omega_{n}^{2}-\omega^{2}}
$$

The modal acoustic pressure amplitude given in Eq. (2.5) can be expressed in a vector form as

$$
\mathbf{a}=\mathbf{Z}_{a}\left(\mathbf{q}+\mathbf{q}_{s}\right)
$$

where $\mathbf{q}$ is the $N$ length modal source strength vector and $\mathbf{q}_{s}=\mathbf{C b}$ is the modal source strength vector due to the virtual membrane at the junction, the third term in the right hand side of Eq. (2.5) representing the acoustic mode coupling due to distortion of the subcavity is accounted in the vector $\mathbf{a} . \mathbf{Z}_{a}$ is the uncoupled acoustic modal impedance matrix. For calculation of acoustic modal characteristics, in the absence of the external force $(\mathbf{q}=\mathbf{0})$, the modal pressure amplitude is given as

$$
\mathbf{a}=\mathbf{Z}_{a} \mathbf{q}_{s}
$$

The vibration amplitude of the $m$-th structural mode in the absence of the external force is expressed as (Kim and Brennan, 1999)

$$
b_{m}(\omega)=\frac{-1}{\rho_{s} h \Lambda_{s}} B_{m}(\omega) \sum_{n=1}^{N} c_{n, m}^{\mathrm{T}} a_{n}(\omega) \quad \Lambda_{s}=\int_{S_{f}} \psi_{m}(\mathbf{r})^{2} d s
$$


where $\rho_{s}$ is density, $h$ is thickness and $\Lambda_{s}$ is area of the vibration membrane. $B_{m}(\omega)$ is the structural mode resonance term. Assuming that damping is negligible

$$
B_{m}(\omega)=\frac{j \omega}{\omega_{m}^{2}-\omega^{2}}
$$

The structural mode resonance term $B_{m}(\omega)$ is unity for the proposed virtual membrane with zero mass and stiffness at the junction. The modal vibration amplitude for the virtual membrane can be expressed in a vector form as

$$
\mathbf{b}=-\mathbf{Y}_{s} \mathbf{g}_{a}
$$

where $\mathbf{g}_{a}=\mathbf{C}^{\mathrm{T}} \mathbf{a}$ is the modal force vector acting on the acoustic system, $\mathbf{Y}_{s}$ is the $M \times M$ diagonal matrix defined as the modal mobility matrix for the virtual membrane, and is given as $\mathbf{Y}_{s}=1 / \Lambda_{s}$.

Equations (2.10) and (2.13) provide modal amplitudes of acoustic pressure in terms of acoustic impedance and the structural modal amplitude of the virtual junction in terms of mobility inside the subcavities. In order to obtain modal characteristics of the irregular cavity, the acoustic and structural impedance as well as mobility of the discretised subcavities are combined. The discretised four bounding domains in Fig. 1b are shown in Fig. 2. Middle subcavities have two membranes, one on its left (L) and right (R) sides, while the end subcavities have one membrane either on left or right side.

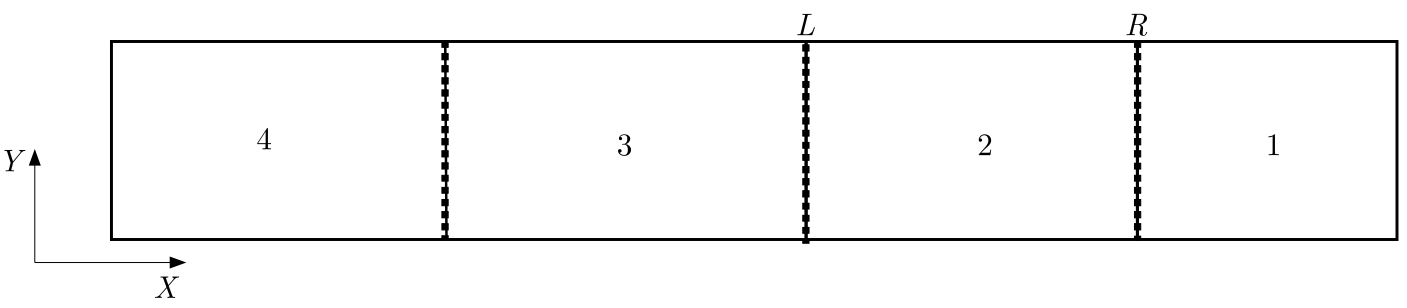

Fig. 2. Rectangular cavity discretized into four regular subcavities

Applying Eqs. (2.10) and (2.13) to each subcavity $k(k=1,2,3,4)$

$$
\begin{aligned}
& \mathbf{a}_{k}= \begin{cases}\mathbf{Z}_{a k}\left(\mathbf{C}_{k} \mathbf{b}_{k}\right) & k=1,4 \\
\mathbf{Z}_{a k}\left(\mathbf{C}_{k}^{L} \mathbf{b}_{K}^{L}+\mathbf{C}_{k}^{R} \mathbf{b}_{K}^{R}\right) & k=2,3\end{cases} \\
& \mathbf{b}_{k}=-\mathbf{Y}_{s k} \mathbf{C}_{k}^{\mathrm{T}} \mathbf{a}_{k} \quad k=1,2,3,4
\end{aligned}
$$

To ensure continuity of velocity on both sides of the membranes, the following equations are imposed

$$
\begin{array}{rlrl}
\mathbf{b}_{k}=\mathbf{b}_{k+1}^{R} & k & =1 \\
\mathbf{b}_{k}^{L}=\mathbf{b}_{k+1}^{R} & k & =2,3 \\
\mathbf{b}_{k-1}^{L}=\mathbf{b}_{k} & k & =4
\end{array}
$$

Above Eqs. (2.14) and (2.15) after simplification and assuming $\lambda=j \omega$ can be rearranged into the following form

$$
\left[\lambda^{2} \mathbf{M}+\lambda \mathbf{L}+(\mathbf{S}+\mathbf{S} \mathbf{1})\right] \mathbf{X}=\mathbf{0}
$$

where $\mathbf{M}, \mathbf{L}, \mathbf{S}$ and $\mathbf{S} \mathbf{1}$ are given below and $\mathbf{X}=\mathbf{a} . \mathbf{S} \mathbf{1}=\mathbf{0}$, for regular cavities. To convert Eq. (2.16) into a standard eigenvalue problem, $Y=\lambda X$ is assumed, and the same is rearranged as

$$
\mathbf{A Z}=\lambda \mathbf{Z}
$$


and

$$
\begin{aligned}
& \mathbf{A}=\left[\begin{array}{cc}
\mathbf{0} & \mathbf{I} \\
-\mathbf{M}^{-1} \mathbf{S} & -\mathbf{M}^{-1} \mathbf{L}
\end{array}\right] \quad \mathbf{Z}=\left\{\begin{array}{l}
X \\
Y
\end{array}\right\} \\
& \mathbf{M}=\left[\begin{array}{ccc}
\Lambda_{s k} \Lambda_{n k} & \cdots & 0 \\
\vdots & \ddots & \vdots \\
0 & \cdots & \Lambda_{s k} \Lambda_{n k}
\end{array}\right] \quad \mathbf{S}=\left[\begin{array}{ccc}
\omega_{n k}^{2} \Lambda_{s k} \Lambda_{n k} & \cdots & 0 \\
\vdots & \ddots & \vdots \\
0 & \cdots & \omega_{n k}^{2} \Lambda_{s k} \Lambda_{n k}
\end{array}\right] \\
& \mathbf{S} 1=\rho_{0} c_{0}^{2}\left[\begin{array}{ccc}
\frac{\Lambda_{s k}}{\Lambda_{n k}} D_{n k, n^{\prime} k} & \cdots & 0 \\
\vdots & \ddots & \vdots \\
0 & \cdots & \frac{\Lambda_{s k}}{\Lambda_{n k}} D_{n k, n^{\prime} k}
\end{array}\right] \quad \mathbf{L}=\rho_{0} c_{0}^{2}\left[\begin{array}{ccc}
C_{k} C_{k}^{\mathrm{T}} & \cdots & 0 \\
C_{k} C_{k-1}^{\mathrm{T}} & C_{k} C_{k}^{\mathrm{T}} & \vdots \\
0 & C_{k} C_{k-1}^{\mathrm{T}} & 0
\end{array}\right]
\end{aligned}
$$

The imaginary values of $\lambda /(2 \pi)$ are the natural frequencies of the irregular cavity.

\section{Results and discussion}

\subsection{Regular subcavity}

A simple two-dimensional rectangular cavity is used to validate the method. The dimensions of the rectangular cavity are length $2 \mathrm{~m}$ and height $1.1 \mathrm{~m}$, as shown in Fig. 3. The dimensions of the regular cavity are taken same for comparison with the IMM method (Anyunzoghe and Cheng, 2002a,b). The number of subcavities is also taken as three for illustration, and can be taken the minimum as two for predicting acoustic modal characteristics.

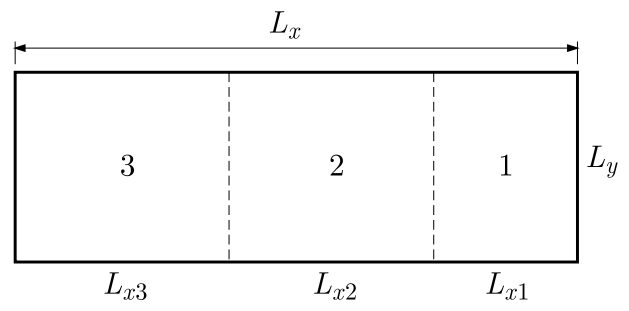

Fig. 3. Rectangular cavity discretized into three subcavities

Five acoustic and structural modes $\left(n_{x}=n_{y}=5, m=5\right)$ of each subcavity are used to predict the acoustic modal characteristics, i.e. natural frequencies and mode shapes of the cavity. The dimensions of the three subcavities are $L_{x_{1}}=0.5 \mathrm{~m}, L_{x_{2}}=0.7 \mathrm{~m}, L_{x_{3}}=0.8 \mathrm{~mm}$ and $L_{y_{1}}=L_{y_{2}}=L_{y_{3}}=1.1 \mathrm{~m}$. The dimensions of the cavities are chosen arbitrarily. The connecting zero mass and stiffness virtual membrane is located at $x_{1}=0.5 \mathrm{~m}$ and $x_{2}=1.2 \mathrm{~m}$. The speed of sound in air is considered as $340 \mathrm{~m} / \mathrm{s}$. The mode shapes for the membrane and bounding cavities are taken as

$$
\psi_{m}(y)=\sin \frac{m \pi y}{L_{y}} \quad \varphi_{n}(x, y)=\cos \frac{n_{x} \pi x}{L_{x}} \cos \frac{n_{y} \pi y}{L_{y}}
$$

Table 1 shows the calculated natural frequencies of the rectangular cavity using the IMCM method. The results obtained from the IMCM method are compared with the exact solution (Morse and Feshbach, 1953) and IMM results (Anyunzoghe and Cheng, 2002a,b). The calculated results are in good agreement with the exact solution and IMM results from the literature. The deviation is less than $3 \%$ at all modes. The proposed IMCM method has less error as compared to the IMM method, for the same number of subcavities and the number of acoustic and structural modes. 
Table 1. Comparison of the predicted natural frequencies of the rectangular cavity with the IMCM

\begin{tabular}{|c|c|c|c|c|c|}
\hline No. & $\begin{array}{c}\text { Mode } \\
\text { order }\end{array}$ & $\begin{array}{c}\text { Exact } \\
\text { solution } \\
{[\mathrm{Hz}]}\end{array}$ & $\begin{array}{c}\text { IMM } \\
{[\mathrm{Hz}]}\end{array}$ & $\begin{array}{c}\text { IMCM } \\
{[\mathrm{Hz}]}\end{array}$ & $\begin{array}{c}\text { Error IMCM } \\
\text { results vs. exact } \\
\text { solution [\%] }\end{array}$ \\
\hline \hline 1 & {$[1,0]$} & 85.7 & 89.4 & 85.5 & 0.23 \\
\hline 2 & {$[0,1]$} & 155.9 & 155.9 & 155.9 & 0 \\
\hline 3 & {$[2,0]$} & 171.5 & 174.0 & 173.8 & 1.34 \\
\hline 4 & {$[1,1]$} & 177.9 & 179.7 & 179.6 & 0.96 \\
\hline 5 & {$[2,1]$} & 231.8 & 233.7 & 234.8 & 1.29 \\
\hline 6 & {$[3,0]$} & 257.25 & 261.8 & 256.8 & 0.17 \\
\hline 7 & {$[3,1]$} & 300.8 & 304.7 & 300.4 & 0.13 \\
\hline 8 & {$[0,2]$} & 311.8 & 311.8 & 311.8 & 0 \\
\hline 9 & {$[1,2]$} & 323.4 & 324 & 323.3 & 0.03 \\
\hline 10 & {$[4,0]$} & 343 & 351.5 & 351.8 & 2.56 \\
\hline
\end{tabular}

Figure 4 shows the pressure mode shapes of mode [1,2] and mode $[2,1]$, respectively, for illustration. The other mode shapes are also in good agreement, however, they are not shown here for brevity. The mode shape shows a good agreement with the exact mode shape except at the subcavity flexible virtual membranes due to the boundary condition, i.e. velocity gradient being zero at the boundary. The difference in the present formulation and the original IMM method is that the IMM method uses the bisection method for calculating natural frequencies, so the subcavity modes are to be differentiated from the true solutions, which makes it difficult to identify the true solution. In the IMCM formulation, the impedance and mobility of the subcavities are converted to a standard eigenvalue problem, hence the solution obtained consists of cavity modes only. Another advantage of this method compared to the IMM method is the formulation being in an impedance and mobility form, which can be easily extended to account for the flexibility of the cavity.
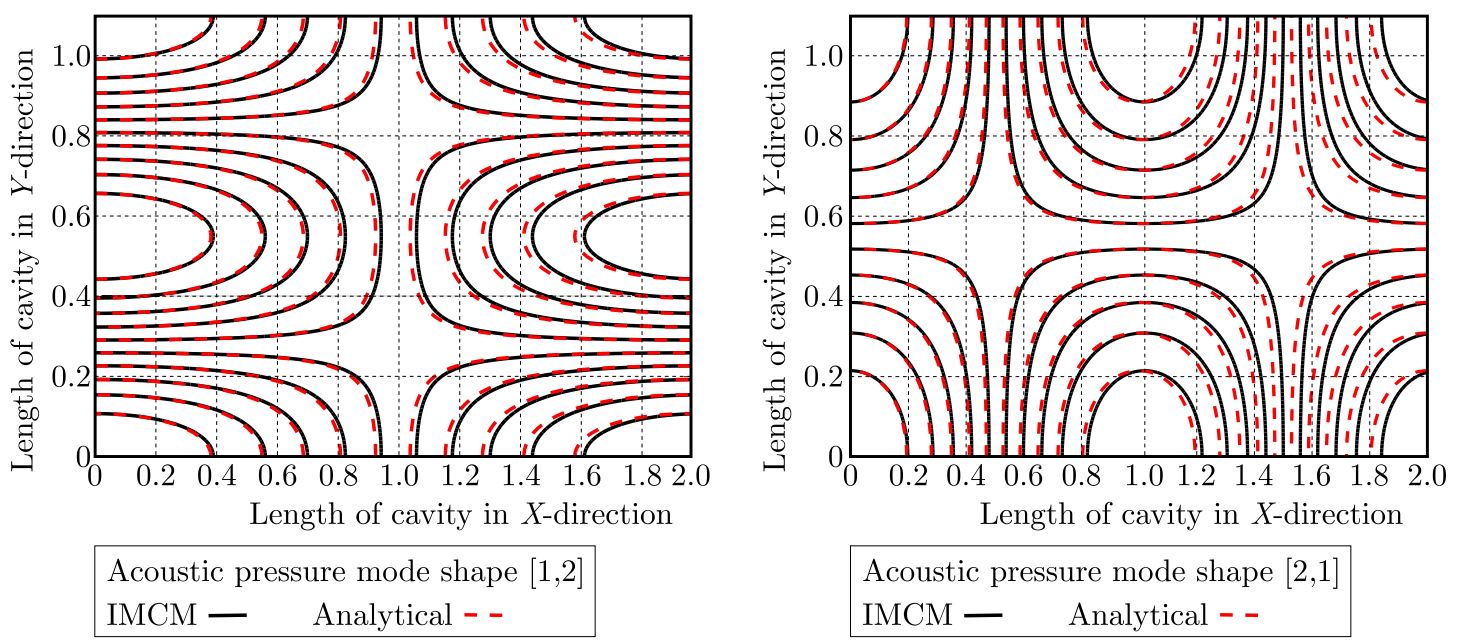

Fig. 4. Acoustic pressure mode shape $[1,2]$ and $[2,1]$ of the rectangular cavity

\subsection{Irregular subcavity}

A flat oval cavity is used to validate the proposed IMCM method. The dimensions of the flat oval cavity are $0.196 \mathrm{~m}, 0.076 \mathrm{~m}$, as shown in Fig. 5. Three subcavities with five acoustic and structural modes $\left(n_{x}=n_{y}=5, m=5\right)$ are used to validate the results. The dimensions of 
the three subcavities are $L_{x_{1}}=0.038 \mathrm{~m}, L_{x_{2}}=0.012 \mathrm{~m}, L_{x_{3}}=0.038 \mathrm{~m}$ and $L_{y_{1}}=L_{y_{2}}=L_{y_{3}}=$ $0.076 \mathrm{~m}$. The connecting zero mass and stiffness virtual membrane is located at $x_{1}=0.038 \mathrm{~m}$ and $x_{2}=0.0158 \mathrm{~m}$. The speed of sound in the air is considered as $340 \mathrm{~m} / \mathrm{s}$. The mode shapes for the virtual membrane and bounding cavities are taken the same as shown in Eqs. (3.1)

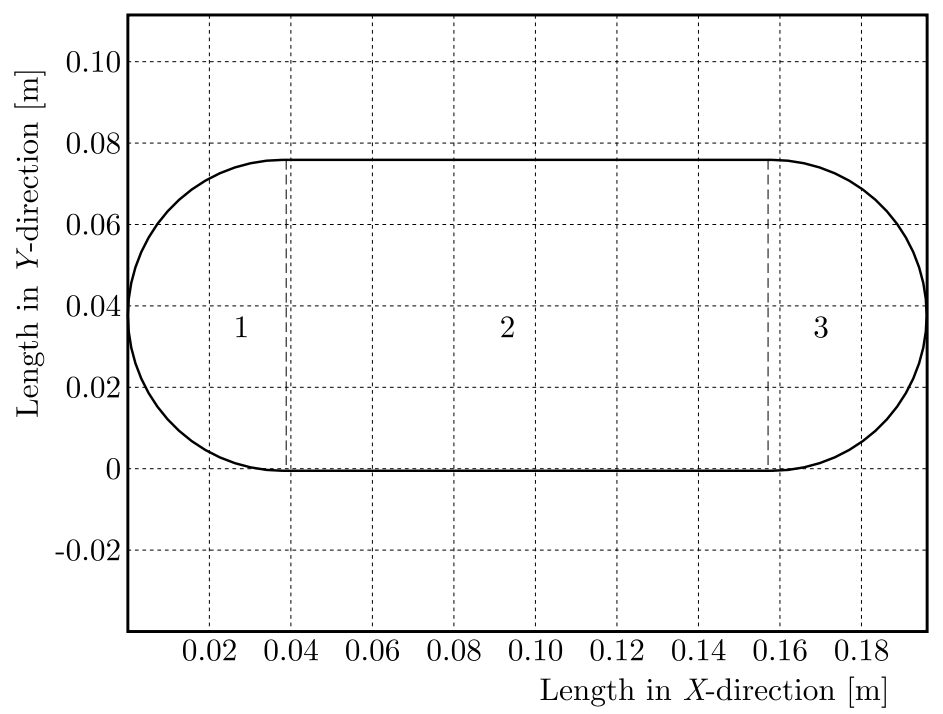

Fig. 5. Flat oval cavity with dimensions and divisions

Table 2 shows the calculated natural frequencies of the flat oval cavity using the IMCM method. The results obtained from the IMCM method are compared with Finite Element (FE) simulated results from ANSYS. ANSYS APDL is used for the FE analysis purpose. The block Lanczos scheme is used for modal analysis.

Table 2. Comparison of the predicted natural frequencies of the flat oval cavity with the IMCM

\begin{tabular}{|c|c|c|c|c|}
\hline No. & $\begin{array}{c}\text { Mode } \\
\text { order }\end{array}$ & $\begin{array}{c}\text { IMCM } \\
{[\mathrm{Hz}]}\end{array}$ & $\begin{array}{c}\text { FEM } \\
{[\mathrm{Hz}]}\end{array}$ & $\begin{array}{c}\text { Error IMCM } \\
\text { vs. FEM [\%] }\end{array}$ \\
\hline \hline 1 & {$[1,0]$} & 945.1 & 956.2 & 1.15 \\
\hline 2 & {$[2,0]$} & 1870.6 & 1892.2 & 1.14 \\
\hline 3 & {$[0,1]$} & 2273 & 2354.5 & 3.46 \\
\hline 4 & {$[1,1]$} & 2650.7 & 2680.9 & 1.12 \\
\hline 5 & {$[3,0]$} & 2828.1 & 2791.0 & 1.32 \\
\hline 6 & {$[2,1]$} & 3245.3 & 3250.1 & 0.15 \\
\hline 7 & {$[3,1]$} & 3630.9 & 3648.1 & 0.47 \\
\hline 8 & {$[0,2]$} & 3934.0 & 3964.2 & 0.76 \\
\hline 9 & {$[1,2]$} & 4489.2 & 4445.3 & 0.99 \\
\hline 10 & {$[4,0]$} & 4673.3 & 4647.7 & 0.55 \\
\hline
\end{tabular}

FLUID29 elements are used with 360 elements, 15 along the circular edge and 24 along width of the cavity. The calculated results match well with the FE results and the deviation is less than $4 \%$ at all modes. Spurious modes are not observed in this method. The pressure mode shapes are shown for modes $[1,0]$ and $[2,1]$ in Fig. 6 and are in a good agreement with the FE mode shapes except at the subcavity flexible virtual membranes due to the boundary condition, i.e. velocity gradient being zero at the boundary. The mode shape contours obtained using the IMCM are shown up to the bounding domain, however, the mode shape contours beyond the actual surface of the irregular cavity need to be discarded. The number of subcavities is arbitrarily selected 
to be three, but it is observed that the convergence of the results to the FE results improves with the increasing number of cavities and the number of acoustic and membrane modes. The convergence of the acoustic natural frequencies with respect to the number of cavities is shown in Table 3, and the convergence with respect to the number of acoustic and membrane modes is shown in Table 4.

(a)
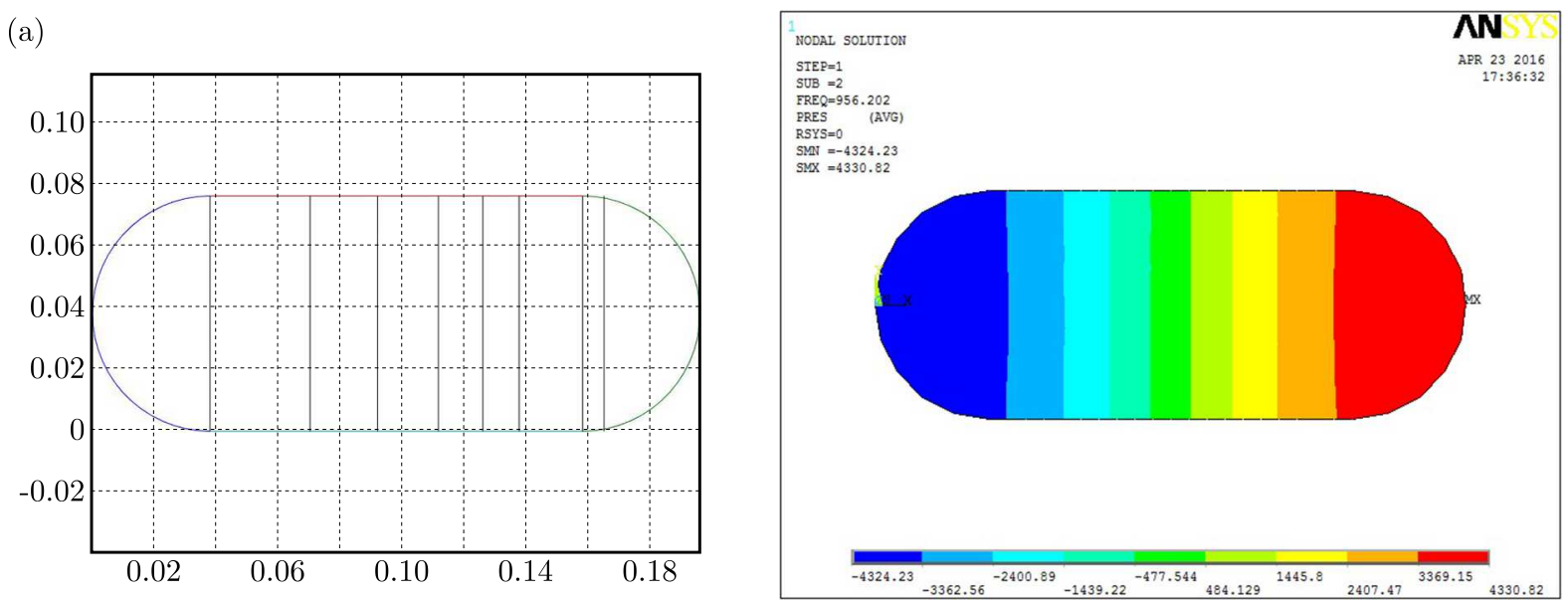

(b)
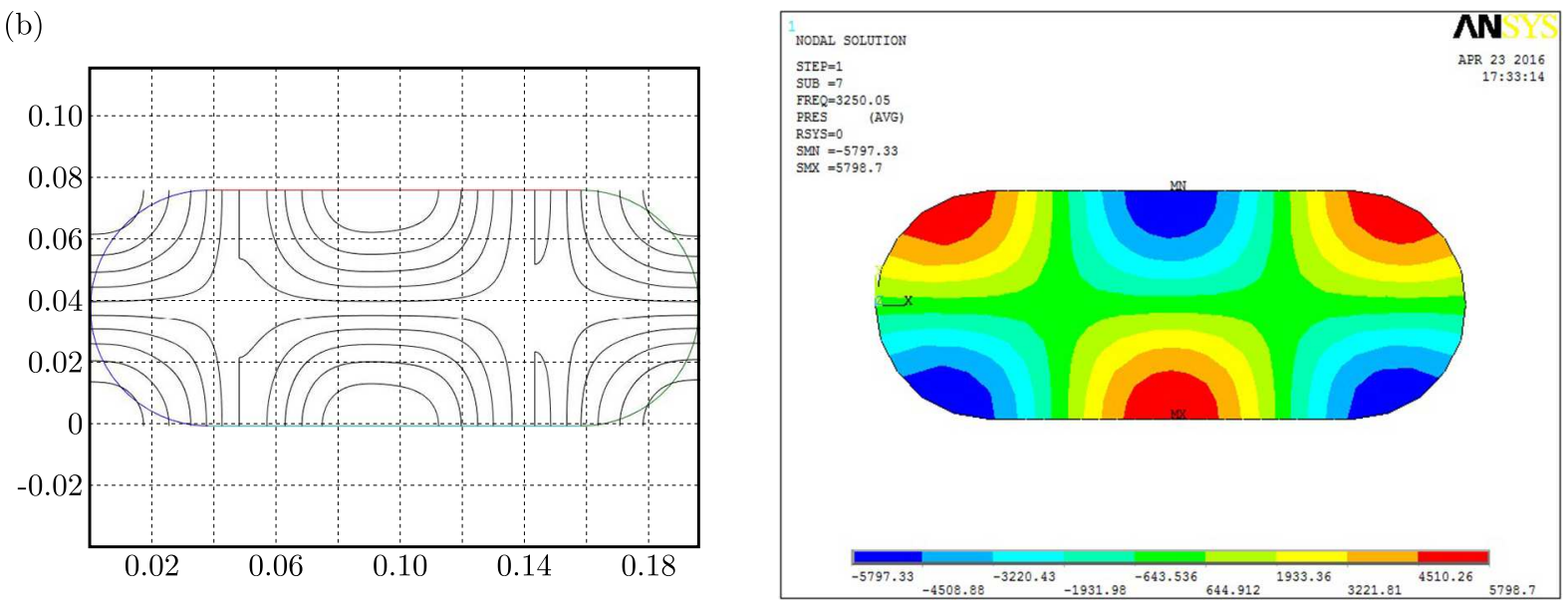

Fig. 6. Comparison of predicted pressure mode shapes $[1,0]$ and $[2,1]$ of the flat oval cavity using the IMCM method with the FEM: left column - IMCM results, right column - numerical results (FEM)

Table 3. Convergence of the predicted natural frequencies of the flat oval cavity with a varying number of subcavities for a constant number of modes $\left(n_{x}=n_{y}=5, m=5\right)$

\begin{tabular}{|c|c|c|c|c|}
\hline Mode & FEM & \multicolumn{3}{|c|}{ IMCM $[\mathrm{Hz}]$} \\
\cline { 3 - 5 } order & {$[\mathrm{Hz}]$} & 3 subcavities & 5 subcavities & 8 subcavities \\
\hline \hline$[1,0]$ & 956.2 & 950.6 & 951 & 958 \\
\hline$[2,0]$ & 1892.2 & 1881 & 1886 & 1894 \\
\hline$[0,1]$ & 2354.5 & 2295 & 2315 & 2343 \\
\hline$[1,1]$ & 2680.9 & 2658 & 2671 & 2683 \\
\hline$[3,0]$ & 2791.0 & 2831 & 2815 & 2804 \\
\hline$[2,1]$ & 3250.1 & 3247 & 3252 & 3257 \\
\hline$[3,1]$ & 3648.1 & 3634.7 & 3643 & 3651 \\
\hline$[0,2]$ & 3964.2 & 3946 & 3952 & 3962 \\
\hline$[1,2]$ & 4445.3 & 4479 & 4468 & 4451 \\
\hline$[4,0]$ & 4647.7 & 4669 & 4655 & 4649 \\
\hline
\end{tabular}


Table 4. Convergence of the predicted natural frequencies of the flat oval cavity with a varying number of acoustic and membrane modes for constant three subcavities

\begin{tabular}{|c|c|c|c|c|}
\hline \multirow{2}{*}{$\begin{array}{c}\text { Mode } \\
\text { order }\end{array}$} & FEM & \multicolumn{3}{|c|}{ IMCM $[\mathrm{Hz}]$} \\
\cline { 3 - 5 } & $n_{x}=n_{y}=5, m=5$ & $n_{x}=n_{y}=9, m=9$ & $n_{x}=n_{y}=12, m=12$ \\
\hline \hline$[1,0]$ & 956.2 & 950.6 & 953 & 954 \\
\hline$[2,0]$ & 1892.2 & 1881 & 1884 & 1889 \\
\hline$[0,1]$ & 2354.5 & 2295 & 2301 & 2315 \\
\hline$[1,1]$ & 2680.9 & 2658 & 2667 & 2673 \\
\hline$[3,0]$ & 2791.0 & 2831 & 2833 & 2835 \\
\hline$[2,1]$ & 3250.1 & 3247 & 3249 & 3253 \\
\hline$[3,1]$ & 3648.1 & 3634.7 & 3639 & 3643 \\
\hline$[0,2]$ & 3964.2 & 3946 & 3951 & 3954 \\
\hline$[1,2]$ & 4445.3 & 4479 & 4481 & 4482 \\
\hline$[4,0]$ & 4647.7 & 4669 & 4672 & 4673 \\
\hline
\end{tabular}

\subsection{Acoustic modal characteristics of off-highway vehicle cabins}

Off-highway vehicles like excavators, tractors and other trolleys have arbitrary shaped cavities, as driver cabins. A simplified two-dimensional model of the cavity is shown in Fig. 7. The acoustic modal characteristics of these cavities are the basic parameters for advanced analysis such as localisation and spaciousness of audio system sound reproduction, identification of acoustic-structural coupling, modal resonances and vibro-acoustic analysis, etc. Typical dimensions of an off high-way vehicle is shown in Fig. 7 with dimensions of $1 \mathrm{~m} \times 1.2 \mathrm{~m}$. The geometry of the cavity is slightly deviated from the rectangular cavity. For applying the proposed IMCM

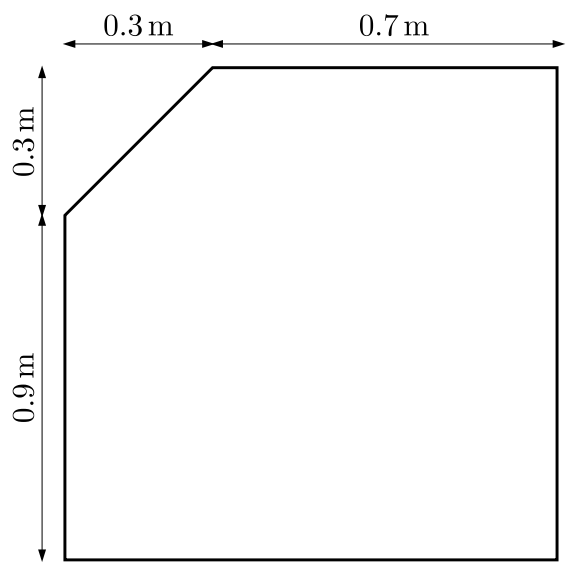

Fig. 7. Geometry of a typical off-highway

method, the overall cavity is divided into three subcavities connected with the virtual membrane. Six acoustic and structural modes $\left(n_{x}=n_{y}=6, m=6\right)$ of each subcavity are chosen to calculate the modal parameters of the irregular cavity. A convergence study has been done by selecting the number of subcavity modes. The dimensions of the three subcavities are $L_{x 1}=0.15 \mathrm{~m}$, $L_{x 2}=0.15 \mathrm{~m}, L_{x 3}=0.7 \mathrm{~m}$ and $L_{y 1}=1.05 \mathrm{~m}, L_{y 2}=L_{y 3}=1.2 \mathrm{~m}$. The connecting zero mass and stiffness virtual membrane is located at $x_{1}=0.15 \mathrm{~m}$ and $x_{2}=0.3 \mathrm{~m}$. The results obtained from the IMCM method are compared with FE simulated results from ANSYS. The calculated modal frequencies match well with the FE results, and the deviation is less than $1.5 \%$ at all modes. It is shown for the first eight modes in Table 5 . The lines of equal sound pressure are calculated for all the modes. The pressure mode shapes are normalised with respect to the maximum value in the 
cavity, and the range is between -1 to 1 in the IMCM method for all the modes. However, for illustration, the 2-nd, 3-rd and 7-th mode shapes of the irregular cavity are compared with the FE mode shapes due to greater complexity. The mode shapes obtained using the IMCM are in a good agreement with the FE mode shapes. The comparison of these mode shapes is shown in Fig. 8. The mode shape of the irregular cavity is accurately predicted by the IMCM method for all the modes. This method can be used for identifying the maximum and minimum resonance points of sound pressure in the cavity, hence, it can be applied to spatial acoustic characteristics analysis and noise control research filed. The irregular cavity mode shapes have more distortions in shape when compared to the regular rectangular cavity, though the geometry is only slightly deviated, signifying a strong interaction of the irregularities in geometry to the mode shapes. These distortions are accurately predicted by the IMCM method.

Table 5. Comparison of the predicted natural frequencies of the irregular cavity with the IMCM

\begin{tabular}{|c|c|c|c|}
\hline No. & IMCM $[\mathrm{Hz}]$ & FE $[\mathrm{Hz}]$ & $\begin{array}{c}\text { Deviation of IMCM } \\
\text { results from the } \\
\text { FE results [\%] }\end{array}$ \\
\hline \hline 1 & 142.9 & 145 & 1.47 \\
\hline 2 & 175.6 & 174.9 & 0.39 \\
\hline 3 & 235.4 & 234.2 & 0.51 \\
\hline 4 & 285.8 & 288.6 & 0.98 \\
\hline 5 & 334.0 & 333.2 & 0.24 \\
\hline 6 & 352.0 & 351.6 & 0.11 \\
\hline 7 & 387.0 & 388.9 & 0.49 \\
\hline 8 & 428.7 & 430.9 & 0.51 \\
\hline
\end{tabular}

\section{Summary}

A new approach has been proposed for computation of acoustic modal characteristics of two-dimensional irregular shaped cavities. The irregular shaped cavities are discretised into regular and irregular subcavities, and the acoustic modal characteristics are estimated via impedance and mobility formulation using multi-connected subcavities with the virtual structural membrane. The proposed method is validated using the rectangular cavity, and the results are compared with the analytical and available literature results. The acoustic modal results are in a good agreement. The proposed method predicts modal characteristics more accurately when compared to other existing methods. The same formulation has been implemented for the flat oval cavity and the results are compared with the numerical results. The results are corroborated, and are in a good agreement. The convergence study has been conducted to understand the number of subcavities and the number of modes participation to get accurate acoustic modal characteristics. The proposed formulation is general and flexible enough to handle different cavity configurations. This method calculates the natural frequencies accurately even when few acoustic modes of the bounding cavities are considered. The proposed method is applied to a practical application like an off-highway vehicle cabin. The proposed method predicts the modal characteristics very accurately, signifying that the proposed method can be applied to spatial acoustic characteristic analysis of two-dimensional irregular cavities which are found in buildings and automobiles. The proposed method can also be applied to the identification of acoustic-structural coupling, modal resonances and vibro-acoustic analysis, where acoustic modal characteristics are essential. In future, the same formulation can be extended to three-dimensional irregular shaped cavities and flexible vibrating boundary surfaces. 
(a)
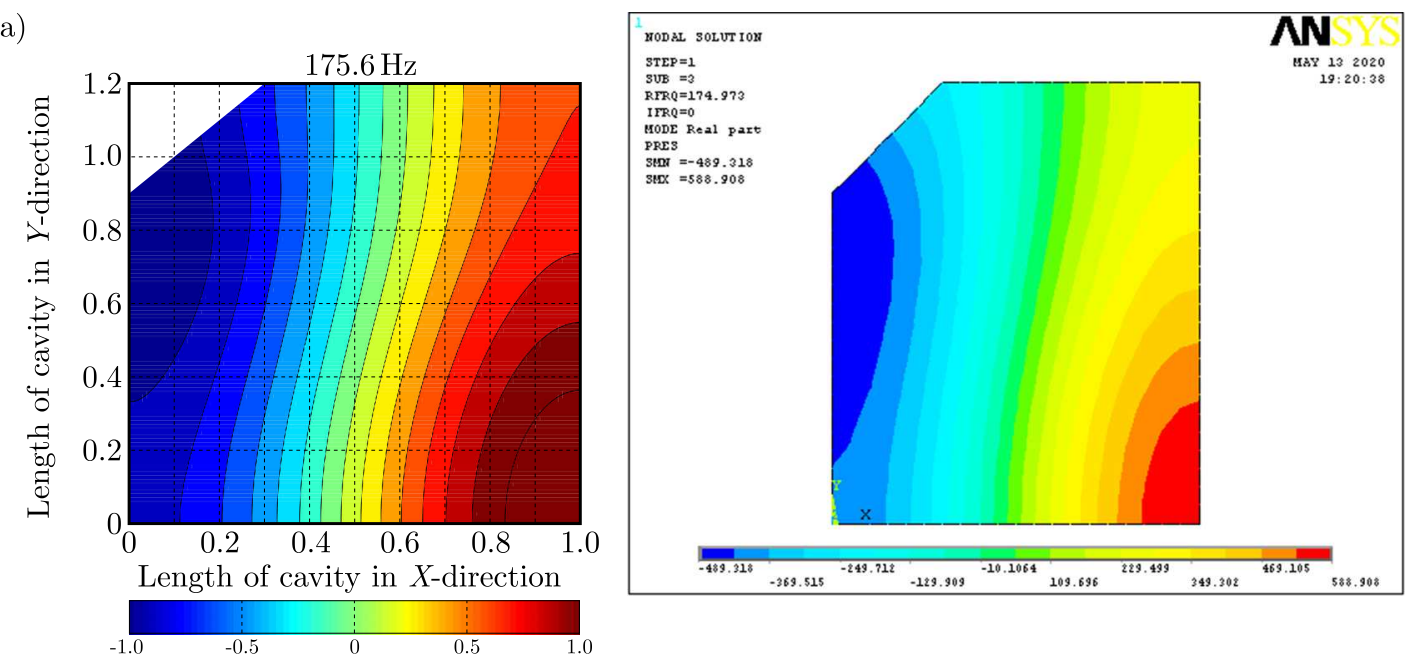

(b)
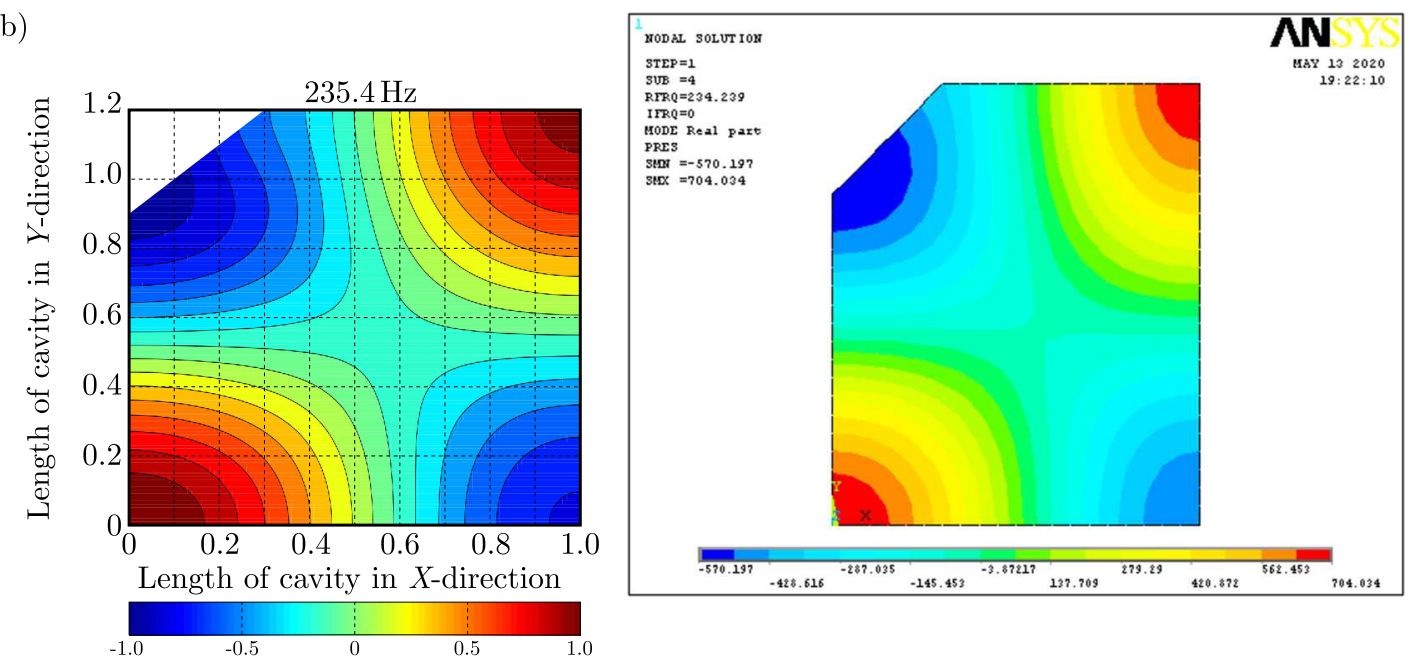

(c)
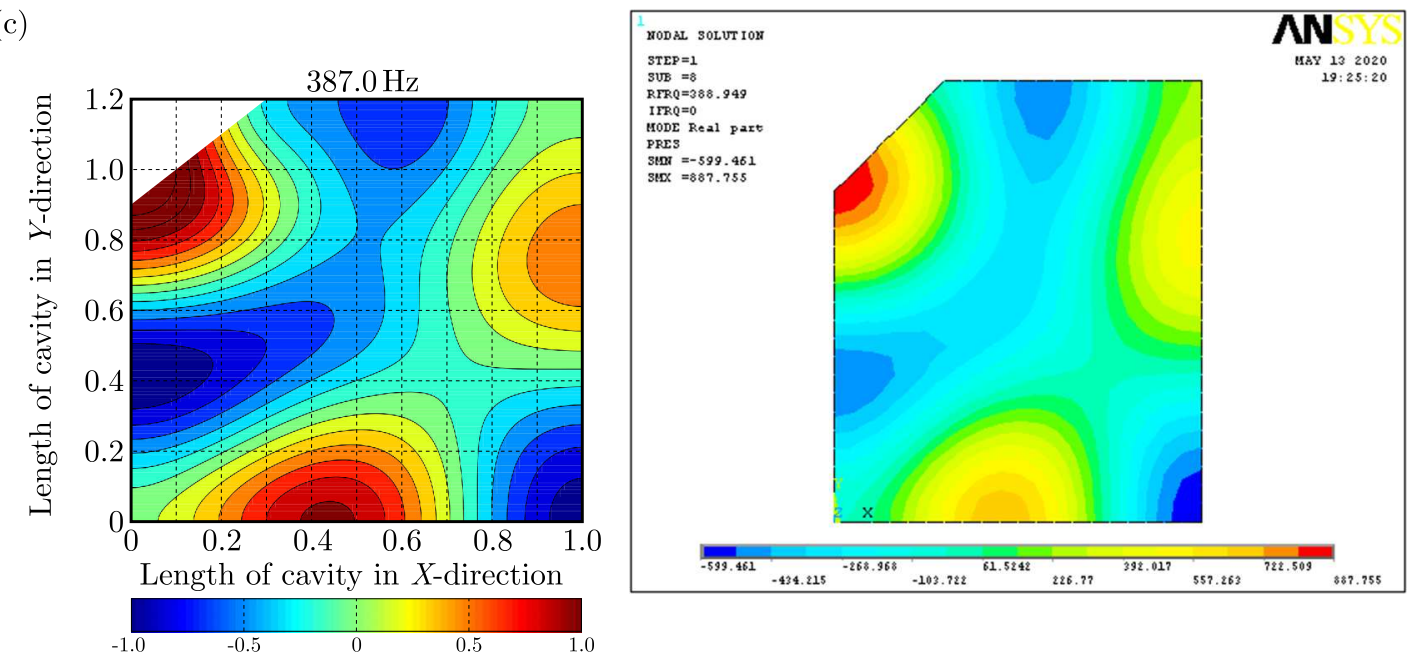

Fig. 8. Comparison of predicted acoustic pressure mode shapes of: (a) 2-nd, (b) 3-rd, (c) 7-th of the irregular cavity using the IMCM method with the FE method 


\section{Acknowledgement}

The authors would like to thank Indian Institute of Technology Hyderabad for providing the required resources for conducting the current research work.

\section{References}

1. Amir N., Starobinski R., 1996, Finding the eigen modes of two dimensional cavities with two axes of symmetry, Acta Acustica united with Acustica, 82, 6, 811-823

2. Anyunzoghe E., Cheng L., 2002a, Improved integro-modal approach with pressure distribution assessment and the use of overlapped cavities, Applied Acoustics, 63, 1233-1255

3. Anyunzoghe E., Cheng L., 2002b, On the extension of the integro-modal approach, Journal of Sound Vibration, 255, 2, 399-406

4. Brebbia A.A., Telles J.C.F., Wrobel L.C., 1984, Boundary Element Techniques, Springer, New York

5. Dowell E.H., Gorman III G.F., Smith D.A., 1977, Acoustoelasticity: general theory, acoustic natural modes and forced response to sinusoidal excitation, including comparison with the experiment, Journal of Sound and Vibration, 52, 519-542

6. Joppa P.D., Fyfe I.M., 1978, A finite element analysis of the impedance properties of irregular shaped cavities with absorptive boundaries, Journal of Sound and Vibration, 56, 61-69

7. KANG S.W., LEE J.M., 2000, Eigenmode analysis of arbitrarily shaped two-dimensional cavities by method of point-matching, The Journal of the Acoustical Society of America, 107, 1153-1160

8. Kim S.M., Brennan M.J., 1999, A compact matrix formulation using the impedance and mobility approach for the analysis of structural-acoustic systems, Journal of Sound Vibration, 223, 1, 97-113

9. Kim Y.Y., KIm D.K., 1999, Applications of waveguide-type base functions for the eigenproblems of two-dimensional cavities, The Journal of the Acoustical Society of America, 106, 1704-1711

10. Missaoui J., Cheng L., 1997, A combined integro-modal approach for predicting acoustic properties of irregular-shaped cavities, The Journal of the Acoustical Society of America, 101, 6, 3313-3321

11. Morse P.M., Feshbach H., 1953, Methods of Theoretical Physics, Vol. II, Mc-Graw Hill, New York

12. PAn J., 1999, A third note on the prediction of sound intensity, The Journal of the Acoustical Society of America, 105, 560-562

13. PAN J., BiEs D.A., 1990, The effect of fluid-structural coupling on sound waves in an enclosure, Theoretical Part, The Journal of the Acoustical Society of America, 87, 2, 691-707

14. Petyt M., Lea J., Koopmann G. H., 1976, A finite element method for determining the acoustic modes of irregular shaped cavities, Journal of Sound and Vibration, 45, 495-502

15. Shi D., Zhang Y., Xiuhai L., 2019, Analysis of acoustic characteristics of arbitrary triangular prism and quadrangular prism acoustic cavities, Shock and Vibration, 2, 1-17

16. Sum K.S., PAN J., 2006, Effects of the inclination of a rigid wall on the free vibration characteristics of acoustic modes in a trapezoidal cavity, The Journal of the Acoustical Society of America, 119, 4, 2201-2210

17. Venkatesham B., Mayank Tiwari, Munjal M.L., 2008, Free vibration analysis of coupled acoustic-structural systems, IISc Centenary - International Conference on Advances in Mechanical Engineering (IC-ICAME) 